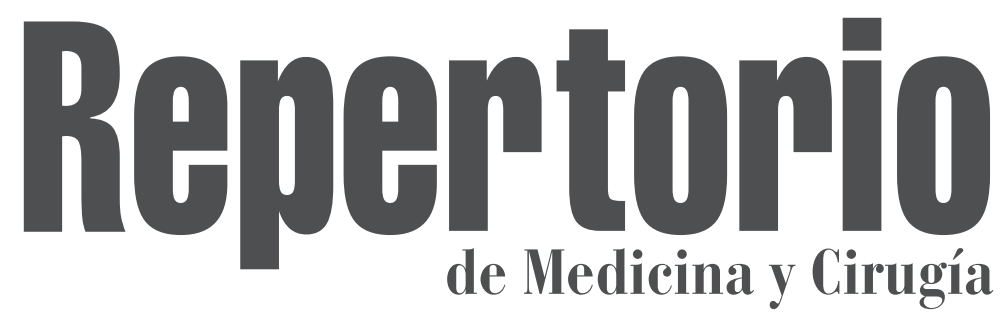

Vol. 280
№2. 2019

ISSN: 0121-7372 • ISSN electrónico: 2462-991X

\title{
Gemelo heterópago
}

\section{Heteropagus Twins}

\section{Jesús Ángel Fernández MD $^{\mathrm{a}}$ \\ Grisel María Marín MD ${ }^{\mathbf{b}}$ \\ Yoadi Elena Tocuyo MD'}

${ }^{a}$ Unidad de Urología Pediátrica, Servicio de Cirugia Pediátrica, Servicio Autónomo Universitario de Maracaibo. Venezuela.

${ }^{b}$ Hospital Servicio de Cirugía Pediátrica, Servicio Autónomo Hospital Universitario de Maracaibo. Venezuela.

\section{R E S U M E N}

Los gemelos parásitos o heterópagos son aquellos unidos asimétricos con partes deformes del parásito que se unen en diferentes regiones del gemelo normal (autosite), del cual depende para su soporte nutricional y crecimiento. Presentamos el caso de un gemelo heterópago con las extremidades inferiores y rudimentos de las superiores del parásito unidas al periné y glúteo derecho del autosite. Aunque no compartían órganos pélvicos, la compresión extrínseca de estructuras óseas del parásito causaron estenosis del recto, lo que obligó a la corrección quirúrgica. Los estudios por imágenes permitieron planificar la cirugía, lo cual facilitó la separación exitosa y lograr la sobrevida del paciente con muy buena calidad de vida. A pesar de ser reportados desde épocas ancestrales, aún generan gran interés debido a la variabilidad en su presentación clínica y al desconocimiento en su etiopatogénesis.

Palabras clave: gemelos heterópagos, gemelos parásitos, gemelos unidos, infección virus del zika.

(C) 2018 Fundación Universitaria de Ciencias de la Salud - FUCS. Este es un artículo Open Access bajo la licencia CC BY-NC-ND (http://creativecommons.org/licenses/by-nc-nd/4.0/).

INFORMACIÓN DEL ARTÍCULO

Historia del artículo:

Fecha recibido: marzo 6 de 2018 Fecha aceptado: septiembre 27 de 2018
Autor para correspondencia. Dr. Jesús Angel Fernández jafernandezf@hotmail.com
DOI

10.31260/RepertMedCir.v28.n1.2019.923 
Heteropagus or "parasitic" twins are asymmetric conjoined twins in whom the tissues of a severely defective twin (parasite) are dependent at variable sites of the other intact twin (autosite) for nutritional supply and growth. Here we present the case of heteropagus twins. The parasite possessed two lower limbs and rudimentary upper limbs attached to the perineum and right buttock of the autosite. Although they did not share pelvic organs, the external compression of the parasite's skeletal structures caused rectum stenosis on the autosite, which required surgical treatment. Imaging studies allowed presurgical planning for the twins' successful separation with good survival and quality of life of the autosite. Although heteropagus twins have been described since ancient times, they still generate great interest due to the varying clinical manifestations and lack of knowledge on their pathogenesis.

Palabras clave: heteropagus twins, parasite twins, conjoined twins, zika virus infection.

(C) 2018 Fundación Universitaria de Ciencias de la Salud - FUCS. This is an open access article under the CC BY-NC-ND license ( ñhttp://creativecommons.org/licenses/by-nc-nd/4.0/).

\section{IN T RODUCCIÓN}

La primera descripción confiable de gemelos unidos parásitos fue realizada en el siglo XVI por Ambroise Paré, cirujano francés que describió un cuerpo acéfalo unido al abdomen de uno de sus pacientes. ${ }^{1}$ La serie más grande publicada que evalúa la incidencia de gemelos heterópagos deriva de una base de datos en Estados Unidos de 7,9 millones de nacimientos registrados durante un período de 8 años en la década de 1970, en la cual se estima que es de 0.05 a 0.1 por 100.000 nacimientos. ${ }^{2}$ Un estudio europeo más reciente en casi 5 millones de neonatos, encontró una incidencia de 0.02 por 100.000 nacimientos. ${ }^{3}$ Ademuyiwa y col. contabilizaron alrededor de 210 casos en una revisión de la literatura hasta el 2013. ${ }^{4}$ Aunque hay una marcada preponderancia femenina en gemelos unidos simétricos (siameses) (72\%), la diferencia por sexo es menos pronunciada en los heterópagos. ${ }^{5}$

Mientras que los gemelos simétricos (siameses) representan fetos simétricamente desarrollados con diferentes sitios de unión anatómica, los heterópagos se caracterizan por uno con defectos graves o incompleto (parásito) unido a cualquier parte del cuerpo del autosite incluso dentro del cuerpo (feto-in-fetu) y dependiente de su gemelo casi intacto (autosite) ${ }^{6}$ El parásito puede encontrarse unido al hipogastrio, región prepúbica epigastrio, cráneo, tórax anterior o sacro del autosite ${ }^{7-9}$ Un reto mayor para el manejo de esta patología se debe a su relativa infrecuencia, lo que se traduce en que los trabajos publicados están basados en reportes de casos aislados. Por ello nos sentimos motivados a divulgar este caso y a presentar nuestra experiencia en el tratamiento de estos gemelos heterópagos.

\section{PRESENTACIÓN DEL CASO}

Neonato masculino de 39 semanas de gravidez producto de tercer embarazo de madre de 28 años y padre de 31 años, sin diagnóstico intrauterino de anomalías congénitas a pesar de control prenatal completo. Fue obtenido por parto vaginal, con peso al nacer de 3,4 kg. El niño tiene 2 hermanos sanos. No había historia de anomalías congénitas familiares ni ingesta de drogas durante el embarazo, sin embargo la madre presentó síntomas de infección por el virus del zika en el primer trimestre del embarazo, sin confirmación por pruebas de laboratorio. El examen físico reveló una gran masa parásita, cuyo pedículo de $11 \mathrm{cms}$ de longitud en sentido anteroposterior y $8 \mathrm{cms}$ de ancho estaba unido al periné, margen anal y glúteo derecho del gemelo intacto (figura 1). La masa parásita estaba formada por dos extremidades inferiores carentes de contractilidad activa, en posición opuesta (imagen en espejo) a los miembros inferiores del autosite, en cada una se identificaron muslos, piernas y pies de configuración cercanos a lo normal, con estructuras óseas de sostén pero movilidad limitada de las articulaciones de rodillas y tobillos. En la cara inferior de la unión de las dos extremidades descritas emergía otra única hacia el extremo proximal pero separada en el extremo distal, con dos esbozos de manos con dedos rudimentarios que corresponderían a las extremidades superiores. En la parte ventral del pedículo de inserción de la masa se evidenció un rudimento de bolsa escrotal, sin gónada en su interior (figura 2). La reconstrucción ósea tomográfica solo mostró presencia de huesos en las extremidades inferiores del parásito, los cuales no se articulaban con las estructuras óseas del autosite (figura 3). La tomografía computadorizada no mostró extensión de la masa parásita a la espina lumbar ni la sacra del autosite. Con excepción de una transposición penoescrotal incompleta, no se demostraron otras anomalías al examen físico en el autosite. El paciente presentó síntomas de obstrucción parcial del recto que mejoraban al movilizar la masa parásita, con salida de evacuaciones. El enema baritado mostró dilatación del marco colónico, mientras que en la tomografía se observó una estructura ósea cercana al margen derecho del ano y porción inferior del recto, que semejaba la cabeza de un fémur del parásito y causaba la obstrucción extrínseca (figura 3). 
La reconstrucción vascular (angioTAC) identificó el origen y el trayecto del pedículo vascular que se originaba en la arteria iliaca común derecha, recorría la pared lateral derecha del recto hasta entrar a la parte central de la masa (figura 4).

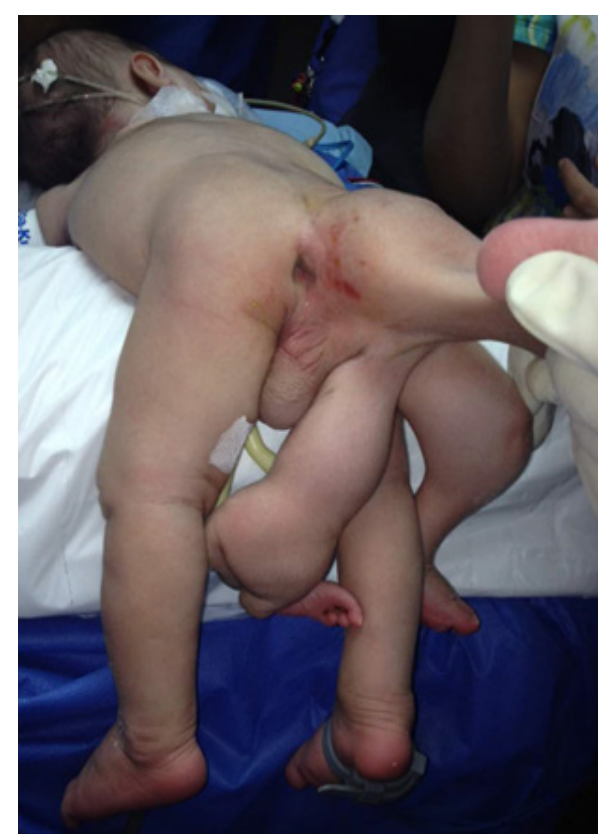

Figura 1. Masa parásita unida al periné, margen anal y glúteo derecho del autosite.

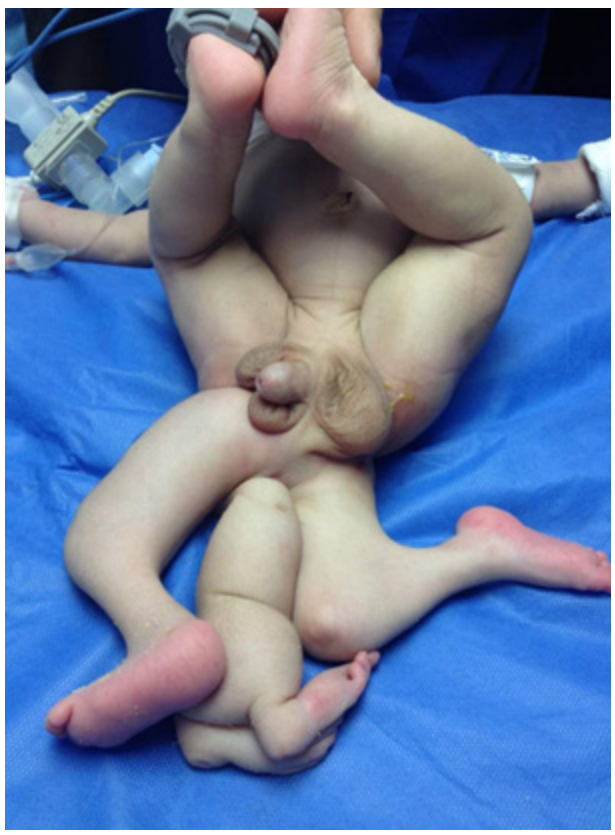

Figura 2. Masa parásita formada por extremidades inferiores, opuestas (imagen en espejo) a los miembros del autosite. En la cara inferior de la unión de estas extremidades, se observa extremidad única que se divide en el extremo distal con esbozos de manos. En la parte ventral del pedículo de inserción, existe un rudimento de bolsa escrotal.

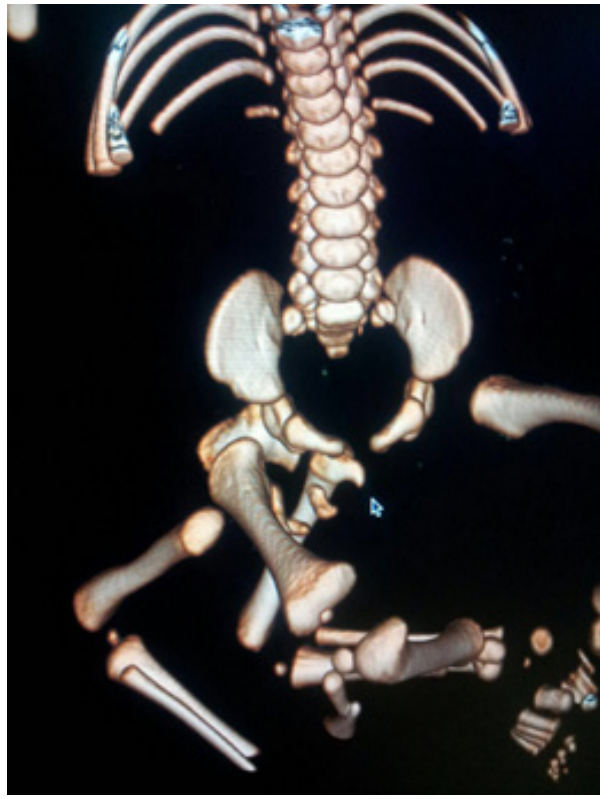

Figura 3. Reconstrucción ósea tomográfica. Se observan estructuras óseas de miembros inferiores de ambos gemelos. No hay articulación ósea del parásito con la pelvis del autosite.

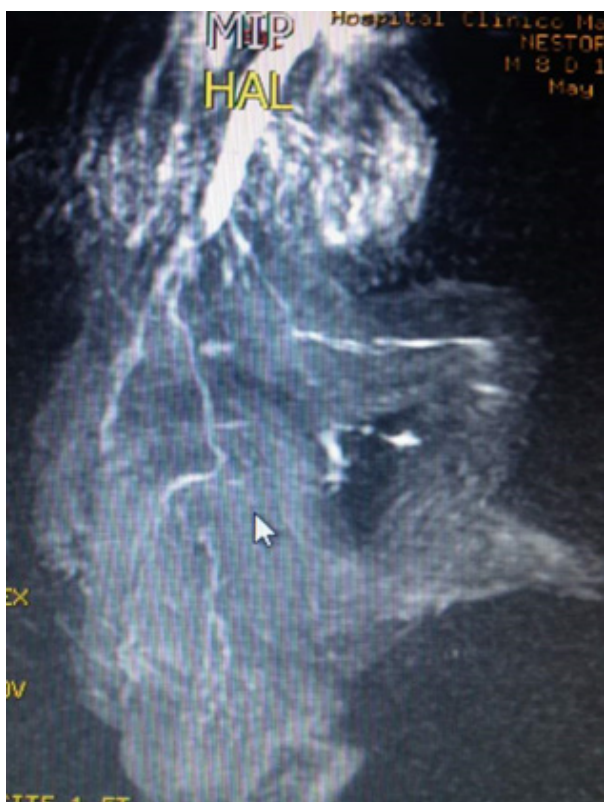

Figura 4. Tomografía abdominopélvica con reconstrucción vascular. Se identifica el origen y el trayecto del pedículo vascular, a partir de la arteria iliaca común derecha (flecha), la cual entra a la parte central de la masa.

La cirugía para la separación de ambos gemelos fue practicada a los 14 días de vida mediante una incisión elíptica en sentido anteroposterior alrededor de la base de la masa. La disección reveló un plano de separación definitivo entre el extremo proximal del fémur izquierdo del parásito, con la pared rectal derecha del extremo distal del recto del paciente. 
Un prominente pedículo neurovascular emergía del lado derecho del recto y entraba a la parte central de la masa. Después de la escisión de la masa el excedente de piel fue modelado hasta obtener un aspecto estético aceptable. Algunos meses posteriores a esta intervención quirúrgica se acentuaron las manifestaciones clínicas de obstrucción rectal. Aunque el ano lucía normoconfigurado, el tacto rectal evidenció una estenosis infranqueable muy cerca del margen anal, lo cual fue confirmado a través de enema baritado (figura 5). El paciente fue sometido a abordaje sagital posterior para corregir dicha estenosis a los 14 meses de la primera cirugía. La evolución clínica del niño desde entonces ha sido satisfactoria y lleva una vida libre de síntomas con desarrollo psicomotor normal. El aspecto estético del sitio operatorio es aceptable (figura 6).

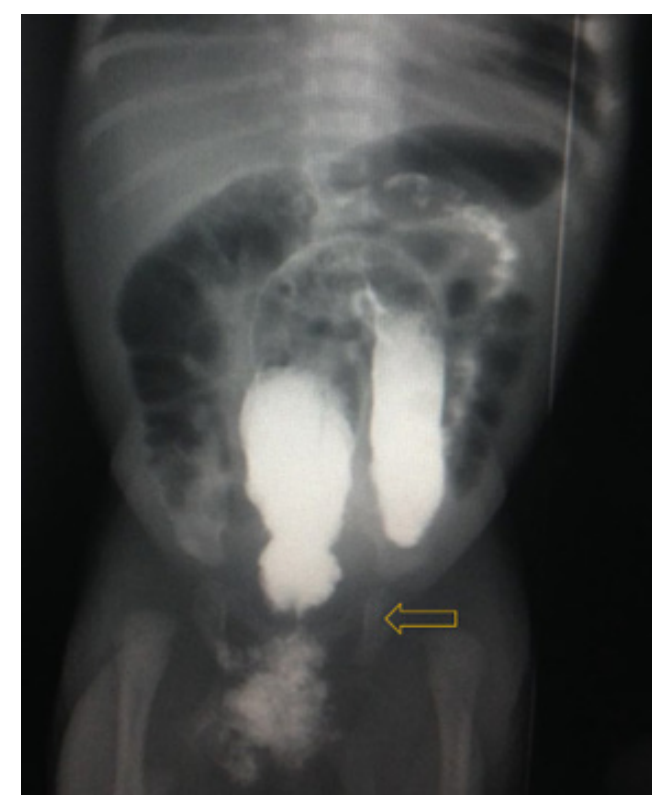

Figura 5. Enema con bario. Se observa disminución de aproximadamente $50 \%$ la luz del extremo inferior del recto (flecha), la cual condiciona dilatación proximal.

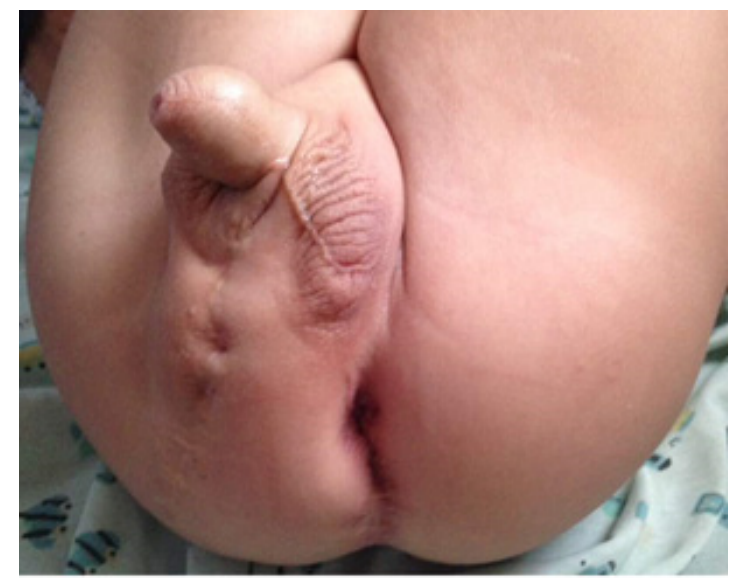

Figura 6. Aspecto posoperatorio mediato. Se observa cicatriz en sitio donde se implantaba la masa.

\section{ISCUSIÓN}

La primera descripción de un miembro accesorio que sobresalía como una masa próxima a la línea media de la región dorsal de un niño fue realizada por Jones y Larkin en 1889. ${ }^{10}$ Algunos nombres fueron dados a esas lesiones: dipodia ipsilateral intraindividual, redundancias heterotópicas ${ }^{11}$ o tripedus. Spencer ${ }^{5}$ reclasificó estos casos como gemelos parásitos y los casos específicos de la región dorsal los llamó raquípagos. Aunque la patogénesis de los gemelos unidos aún es un misterio, se ha tratado de explicar a través de algunas teorías entre otras la de la fusión, según la cual la ocurrencia de la anomalía es determinada al final de la segunda semana de gestación cuando dos gemelos heterocigotos se fusionan para formar una estructura embrionaria común que contiene dos discos embrionarios en un saco vitelino único ${ }^{12}$ y la teoría de la fisión, la cual establece que la división incompleta del embrión seguida de fusión subsecuente a los 14 o 15 días de la fertilización, resulta en gemelos unidos. ${ }^{12}$ Con relación a la patogénesis de la asimetría entre ambos gemelos, Donitz y col. señalan que se debe al compromiso vascular causante de que los tejidos del gemelo parásito se vuelvan dependientes de las colaterales derivadas del autosite. ${ }^{13}$ Con respecto a la anatomía y morfología de los gemelos parásitos, esta varía de acuerdo con el sitio de implantación en el autosite; aparecen extremidades superiores con sus remanentes óseos cuando la unión parásita se produce en la región cervical o torácica, mientras que miembros inferiores con sus huesos rudimentarios predominan en los casos de implantación dorsal baja y lumbar. ${ }^{14}$ En el caso reportado la masa parásita simuló rudimentos de extremidades superiores entre dos inferiores de configuración cercana a lo normal. En cuanto a las anomalías asociadas en el autosite, por lo general son vecinas o están en el sitio de unión, de tal forma que en gemelos raquípagos, las anomalías más comunes se localizan en la columna vertebral y/o el sistema nervioso central $^{12,15,16}$, mientras que las malformaciones cardiacas congénitas se asocian con los parásitos onfalópagos. ${ }^{5}$ Se destaca el hecho de que la madre presentó síntomas compatibles con infección del virus del zika en el primer trimestre del embarazo; sin embargo no hubo confirmación por laboratorio. No obstante, se hizo una revisión de la literatura sobre la relación entre la infección por este virus y gemelos unidos, sin conseguir reportes al respecto, encontrándose sólo múltiple evidencia de anomalías neurológicas y oftálmicas producidas por el zika. ${ }^{17}$ En el caso reportado el autosite presentó obstrucción parcial sintomática del extremo inferior del recto la cual mejoraba con la movilización de la masa parásita, por lo que al inicio se pensó que dichos síntomas desaparecerían con la cirugía, sin embargo persistieron, lo que obligó a la exploración a través de abordaje sagital posterior, momento en el que se evidenció disminución de cerca de $50 \%$ de la circunferencia del recto, $1 \mathrm{~cm}$ por encima de la línea pectínea. A pesar de que la estenosis rectal es un desorden del espectro de malformaciones anorectales descritas 
por el grupo del Dr. Alberto Peña ${ }^{18}$, particularmente asociadas con masa presacra, se piensa que la lesión fue producida por la compresión extrínseca del fémur del parásito sobre el recto.

El diagnóstico prenatal para detectar gemelos heterópagos intraútero juega un papel importante para guiar el subsecuente manejo obstétrico y quirúrgico, así como en la preparación psicológica de los padres para limitar el impacto emocional al momento del nacimiento. ${ }^{12}$ Como ocurrió con nuestro paciente, es lamentable que hayan muchos ejemplos en los cuales falló el diagnóstico prenatal de gemelos heterópagos. ${ }^{19-21}$ Debido a la infrecuencia es poco probable que todos los cirujanos tengan experiencia individual en el tratamiento de estos casos, por lo que la planificación preoperatoria y el abordaje quirúrgico debe elaborarse en forma individual para cada uno, sobretodo si el parásito comparte órganos con el autosite. La descripción de las técnicas quirúrgicas específicas en gemelos heterópagos es limitada, dada las variaciones en conexiones vasculares, óseas y de tejidos blandos entre autosite y parásito. ${ }^{12}$ La tomografía computadorizada, el ultrasonido y las imágenes de resonancia magnética representan los estudios más utilizados en todos los tipos de gemelos unidos. ${ }^{12}$ La angiotomografía computadorizada por lo general permite delinear la anatomía vascular entre ambos fetos, a pesar de que la descripción del pedículo vascular del gemelo heterópago no siempre se reporta. ${ }^{12,22}$ En este caso la tomografía computadorizada con reconstrucción ósea y vascular permitió conocer la relación del gemelo parásito con los órganos pélvicos del autosite y la identificación del origen y trayecto del pedículo vascular del parásito, lo que favoreció la sobrevida y expectativa de vida normal en el paciente, portador de una entidad clínica con una mortalidad reportada hasta en el $31 \%$ de los casos. ${ }^{12}$ La ecocardiografía debe solicitarse a los toracópagos y onfalópagos, quienes presentan anormalidades cardiacas en el autosite entre 25 y $39 \% .^{5}$

\section{CONCLUSIONES}

Los gemelos unidos representan una entidad poco común, susceptible de ser tratada con éxito para garantizar la sobrevida con una calidad de vida aceptable. Deben hacerse esfuerzos por publicar todos los casos con una descripción minuciosa de las anomalías asociadas, la anatomía de la lesión y el procedimiento quirúrgico empleado para ganar experiencia en el estudio de esta entidad.

\section{REFEREN CIAS}

1. Pare A. On Monsters and Marvels. United State: University of Chicago Press; 1995.

2. Edmonds LD, Layde PM. Conjoined twins in the united states, 1970-1977. Teratology. 1982;25(3):301-8. doi: 10.1002/ tera.1420250306.
3. Martinez-Frias ML, Bermejo E, Mendioroz J, Rodriguez-Pinilla E, Blanco M, Egues J, et al. Epidemiological and clinical analysis of a consecutive series of conjoined twins in Spain. Journal of pediatric surgery. 2009;44(4):811-20. doi: 10.1016/j.jpedsurg.2008.07.002.

4. Ademuyiwa AO, Alabi EO, Idiodi-Thomas HO, Bankole OB, Elebute OA, Alakaloko FM, et al. Surgical management of pygopagus parasiticus in a developing country: Challenges and review of the literature. Journal of Pediatric Surgery Case Reports. 2015;3(2):5862. doi: 10.1016/j.epsc.2014.12.003

5. Spencer R. Parasitic conjoined twins: external, internal (fetuses in fetu and teratomas), and detached (acardiacs). Clin Anat. 2001;14(6):428-44. doi: 10.1002/ca.1079

6. Poradowska W, Jaworska M, Reszke S, Lodzinski K. Conjoined twins and twin parasite: clinical analysis of three examples. Journal of pediatric surgery. 1969;4(6):688-93.

7. O'Neill JA, Jr., Holcomb GW, 3rd, Schnaufer L, Templeton JM, Jr., Bishop HC, Ross AJ, 3rd, et al. Surgical experience with thirteen conjoined twins. Annals of surgery. 1988;208(3):299-312.

8. Chadha R, Bagga D, Dhar A, Mohta A, Malhotra CJ, Taneja SB. Epigastric heteropagus. Journal of pediatric surgery. 1993;28(5):723-7.

9. Ozcan C, Ergun O, Guclu C, Tumuklu M, Alper H, Erdener A. An unusual case of epigastric heteropagus: parasite with a rudimentary heart. Journal of pediatric surgery. 2000;35(10):1523-5.

10. Jones R, Larkin FC. Removal of Accessory Limb and Meningocele from the Back of a Child, and its Anatomy. British medical journal. 1889;2(1493):310-1.

11. O'Rahilly R. Morphological patterns in limb deficiencies and duplications. The American journal of anatomy. 1951;89(2):135-93.

12. Sharma G, Mobin SS, Lypka M, Urata M. Heteropagus (parasitic) twins: a review. Journal of pediatric surgery. 2010;45(12):2454-63. doi: 10.1002/aja.1000890202

13. Kapur V, Kulkarni M, Shenoy M. Asymmetric conjoined twins. Pediatr Surg Int. 1997;12:308-9.

14. Ratan SK, Rattan KN, Magu S, Rohilla S, Purwar P, Mathur SK. Thoracolumbar rachipagus parasite. Pediatric Surgery International. 2004;20(4):298-300.

15. Spencer R. Rachipagus conjoined twins: they really do occur! Teratology. 1995;52(6):346-56.

16. Nanni L, Perrelli L, Velardi F. Accessory lower limb in a newborn with multiple malformations. European journal of pediatric surgery : official journal of Austrian Association of Pediatric Surgery [et al] = Zeitschrift fur Kinderchirurgie. 1994;4(1):51-3. doi: 10.1055/s-2008-1066068

17. Platt DJ, Miner JJ. Consequences of congenital Zika virus infection. Current opinion in virology. 2017;27:1-7. doi: 10.1016/j. coviro.2017.09.005.

18. Hamrick M, Eradi B, Bischoff A, Louden E, Pena A, Levitt M. Rectal atresia and stenosis: unique anorectal malformations. Journal of pediatric surgery. 2012;47(6):1280-4.

19. Cury EK, Schraibman V. Epigastric heteropagus twinning. Journal of pediatric surgery. 2001;36(7):E11.

20. Hager J, Sanal M, Trawoger R, Gassner I, Oswald E, Rudisch A, et al. Conjoined epigastric heteropagus twins: excision of a parasitic twin from the anterior abdominal wall of her sibling. European journal of pediatric surgery : official journal of Austrian Association of Pediatric Surgery [et al] = Zeitschrift fur Kinderchirurgie. 2007;17(1):66-71. doi: 10.1055/s-2007-964951

21. Husain AN, Muraskas J, Lambert G, Dado D, Lynch J. Parasitic conjoined twins with omphalocele and tetralogy of Fallot. Pediatric pathology. 1989;9(3):321-8.

22. Trainavicius K, Kazlauskas V, Gurskas P. Epigastric heteropagus conjoined twins. Journal of Pediatric Surgery Case Reports. 2013;1(5):114-7. doi: 10.1016/j.epsc.2013.03.012 